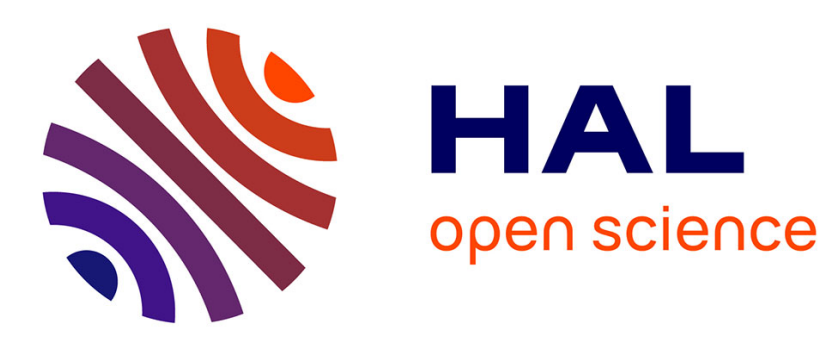

\title{
La voyelle inaccentuée "e" en position initiale: analyses acoustiques et enjeux pédagogiques pour l'anglais L2
}

\author{
Anne Tortel, Sophie Herment
}

\section{To cite this version:}

Anne Tortel, Sophie Herment. La voyelle inaccentuée "e" en position initiale: analyses acoustiques et enjeux pédagogiques pour l'anglais L2. XXXIIe Journées d'Études sur la Parole, Jun 2018, Aix-enProvence, France. 10.21437/JEP.2018-67 . hal-01811589

\author{
HAL Id: hal-01811589 \\ https://hal.science/hal-01811589
}

Submitted on 4 Jul 2018

HAL is a multi-disciplinary open access archive for the deposit and dissemination of scientific research documents, whether they are published or not. The documents may come from teaching and research institutions in France or abroad, or from public or private research centers.
L'archive ouverte pluridisciplinaire HAL, est destinée au dépôt et à la diffusion de documents scientifiques de niveau recherche, publiés ou non, émanant des établissements d'enseignement et de recherche français ou étrangers, des laboratoires publics ou privés. 


\title{
La voyelle inaccentuée $<\mathrm{e}>$ en position initiale : analyses acoustiques et enjeux pédagogiques pour l'anglais $\mathbf{L 2}$.
}

\author{
Anne Tortel \& Sophie Herment \\ Aix-Marseille Université, Laboratoire Parole et Langage, CNRS-UMR7309, \\ 5 avenue Pasteur, 13100 Aix en Provence, France \\ anne.tortelduniv-amu.fr; sophie.herment@univ-amu.fr
}

\section{RESUME}

Les travaux sur la réduction vocalique sont nombreux mais les études $d u<e>$ inaccentué à l'initial de mot sont quasi inexistantes, notamment lorsqu'il s'agit d'étudier des productions d'apprenants francophones. L'étude de cette voyelle est donc ici réalisée sur un corpus d'anglais oral lu par 10 natifs anglophones et 20 apprenants francophones de l'anglais en analysant les valeurs de F1-F2 et de durées. Les résultats révèlent un changement phonologique en cours intéressant pour l'anglais RP contemporain et les données obtenues avec nos trois groupes de locuteurs permettent de comprendre la distribution et la réalisation de la voyelle inaccentuée $<\mathrm{e}>$ par les natifs et les apprenants de l'anglais. Cette étude nous permet également d'établir des perspectives pédagogiques pour l'apprentissage et l'enseignement de l'anglais L2.

\section{ABSTRACT}

Unstressed vowel $<\mathrm{e}>$ in initial position: acoustic analyses and pedagogical issues for ESL.

Vocalic reduction has been studied in various phonetic and phonological perspectives but the realisation of reduced $\langle\mathrm{e}\rangle$ in initial syllables in English has hardly been dealt with. In this study the realisations of this unstressed vowel produced by 10 native English speakers and 20 French learners of English were analysed using an automatic extraction of F1-F2 values and durations in a corpus of read speech. The results are enlightening as far as phonological change is concerned and the data obtained with the three groups of speakers contribute to understanding the distribution and realisation of unstressed vowels by native speakers and learners of English. This study finally opens on pedagogical perspectives for both learning and teaching ESL.

MOTS-CLES : réduction vocalique, rythme, durée vocalique, apprenants, anglais L2.

KEYWORDS: vocalic reduction, rhythm, vocalic duration, learners, L2 English.

\section{Introduction}

L'acquisition du rythme de l'anglais représente une des difficultés majeures de l'apprentissage de cette langue pour des francophones et tout particulièrement la production des voyelles inaccentuées . A un niveau avancé, les apprenants francophones de l'ang lais réalisent souvent très bien les voyelles accentuées, mais pèchent à prononcer correctement les voyelles inaccentuées, surtout lorsqu'elles sont réduites (Tortel, 2009). Cela vient du fait que les deux langues présentent une organisation rythmique complètement différente (Wenk \& Wioland, 1982). En anglais l'alternance de temps forts et de temps faibles a pour conséquence sur le système vocalique la réduction de certaines 
voyelles (Bolinger, 1981). Cette caractéristique semble typique de l'anglais oral et non du français (même si les réalisations des voyelles françaises varient sur le plan phonétique, voir Gendrot \& Adda-Decker, 2007). Alors qu'il existe des règles régissant le processus de réduction en anglais, cela reste très difficile pour un apprenant de savoir quelle voyelle il doit réaliser lorsqu'il produit une réduction. En effet, la voyelle d'une syllabe inaccentuée peut avoir sa forme pleine ou sa forme réduite, et lorsqu'elle est réduite, plusieurs réalisations sont parfois possibles. Tel est le cas, par exemple, du mot enjoy pour lequel il est possible de prononcer [i, e, ə] (Wells, 2008). Dans une étude fondée sur les données des dictionnaires de prononciation anglaise Longman Pronunciation Dictionary (Wells, 2008) et English Pronunciation Dictionary (Jones, 2011), désormais LPD3 et EPD17, Herment (2010) montre que des indices permettent de prédire les réalisations des voyelles réduites en positions médiane et finale, mais que très peu de tendances se dégagent pour la position initiale, et particulièrement en ce qui concerne le graphème $<\mathrm{e}>$. De nombreuses combinaisons de variantes apparaissent dans les deux dictionnaires, qui ne sont pas toujours en accord : pour ne prendre qu'un exemple, la première syllabe du mot emotion se prononce [i] ou [ə] selon LPD3 et [I] selon EPD18 ( $c f$. tableau 1). Nous proposons donc de nous intéresser dans un premier temps à la réalisation de la voyelle $\langle\mathrm{e}\rangle$ en position initiale inaccentuée dans un corpus de parole lue par des locuteurs natifs, afin de comparer nos résultats aux données dictionnairiques et de tenter d'établir des tendances robustes pour la réalisation de cette voyelle. Dans un second temps, nous examinons les réalisations de cette voyelle par deux groupes d'apprenants francophones sur le même co rpus lu afin de les comparer aux productions des natifs et de montrer quelles sont les enjeux pédagogiques que l'on peut en retirer.

\section{Corpus}

\subsection{AixOx}

Les recherches présentées ici ont été réalisées à partir du corpus multilingue AixOx (Herment et al., 2012, 2014). Ce corpus fait partie de la base de données OMProDat (Open Multilingual Prosodic Database, Hirst et al., 2013), qui constitue une collection d'enregistrements suivant le protocole Eurom 1 (Chan et al., 1995) dans plusieurs langues. AixOx est composé de productions orales enregistrées en chambre sourde par des natifs anglophones et francophones, des apprenants anglophones du français et des apprenants francophones de l'anglais. 40 textes composés de 5 phrases chacun et racontant des histoires du quotidien ont été lues par les locuteurs. Pour les besoins de cette étude, seules les productions des natifs anglophones et des apprenants francophones de l'anglais ont été analysées. Cela représente plus de 20 heures de parole lue pour 30 locuteurs : 10 locuteurs natifs (5 hommes, 5 femmes) âgés de 18 à 26 ans, tous originaires d'Oxford et y résidant, et parlant une variété d'anglais qualifiée de "RP contemporain» selon les experts de la British Library $^{1}$; 10 apprenants francophones de l'anglais (5 femmes, 5 hommes) de niveau C (expérimenté) selon le CECRL ${ }^{2}$, âgés de 18 à 32 ans, originaires de la région PACA ; 10 apprenants francophones de l'anglais de niveau $\mathrm{B}$ (indépendant) selon le CECRL, âgés de 18 à 32 ans, 8 d'entre eux étant de la région PACA et 2 originaires de la région parisienne. 36 mots du corpus contiennent la voyelle $<\mathrm{e}>$ à l'initiale de syllabe. Parmi eux, 3 apparais sent à plusieurs reprises soit dans le corps

1 http://www.bl.uk/learning/langlit/sounds/find-out-more/received-pronunciation/, consulté le 25 janvier 2018. RP signifie «Received Pronunciation». Il s'agit de la variété britannique standard.

2 Cadre Européen Commun de Référence pour les Langues, Unité des politiques linguistiques de Strasbourg, www.coe.int/lang-CECR, consulté le 5 février 2018 
d'un même texte, comme pour department ( 2 fois) et delivery ( 2 fois), soit dans des textes différents, comme pour department (apparaissant 6 fois au total) ou encore because ( 2 fois). Cela donne un total de 46 occurrences par locuteur.

\subsection{Donné es dictionnairiques et hypothèse}

Pour les 36 mots du corpus, les prononciations $\mathrm{du}<\mathrm{e}>$ présent dans la syllabe initiale ont été relevées dans les deux dictionnaires mentionnés plus haut (LPD3 \& EPD18). Le tableau 1 en montre un extrait pour les premiers mots. On note une grande variabilité, et le manque de consensus entre les deux ouvrages est frappant. La possibilité est donnée par LPD3 de prononcer un schwa [ə] pour toutes les occurrences, ce qui n'est pas le cas de EPD18. D'un point de vue diachronique, la confrontation avec les données de 1967 (Jones, 19673) est parlante : pour la plupart des mots étudiés, [ə] n'est pas donné comme une variante possible. Nous formulons donc l'hypothèse que les locuteurs de RP contemporain montrent une tendance à la centralisation des voyelles initiales inaccentuées et à prononcer [ə].

\begin{tabular}{|c|c|c|c|}
\hline Words & LPD3 & EPD18 & Jones 1967 \\
\hline Because & {$[i$, ə] } & {$[\mathrm{I}, \partial]$} & {$[i$, ə] } \\
\hline Behind & {$[i$, ə] } & {$[\mathrm{I}, \partial]$} & {$[\mathrm{i}]$} \\
\hline Believe & {$[i, \partial]$} & {$[\mathrm{I}$, , ] } & {$[\mathrm{i},(\partial)]$} \\
\hline Behave & {$[i$, ə] } & {$[\mathrm{I}, \partial]$} & {$[i,(\partial)]$} \\
\hline Department & {$[i$, ə] } & {$[\mathrm{I}, \partial]$} & [i] \\
\hline Delivered & {$[i$, o] } & {$[\mathrm{I}$, , ] } & [i] \\
\hline Delivery & {$[i$, ə] } & {$[\mathrm{I}$, ə] } & {$[\mathrm{i}]$} \\
\hline December & {$[i$, ə] } & {$[\mathrm{I}$, ə] } & {$[\mathrm{i}, \mathrm{i}:]$} \\
\hline Devoured & {$[i$, o] } & {$[\mathrm{I}$, ə] } & [i] \\
\hline Destroyed & {$[i$, o] } & {$[\mathrm{I}$, ə] } & [i] \\
\hline Dependent & {$[i$, , $]$} & {$[\mathrm{I}, \partial]$} & [i] \\
\hline Effect & {$[\partial, \mathrm{i}]$} & {$[\mathrm{I}]$} & [i] \\
\hline Electric & {$[i, \partial]$} & [i] & {$[\mathrm{i},(\partial)]$} \\
\hline Emergency & {$[i$, ə] } & {$[\mathrm{I}, \mathrm{i}:]$} & [i] \\
\hline Emotion & {$[i$, ə] } & {$[\mathrm{I}]$} & [i] \\
\hline Enjoyed & {$[\mathrm{i}, \mathrm{e}$, ə] } & {$[\mathrm{I}, \partial]$} & {$[\mathrm{i},(\mathrm{e})]$} \\
\hline
\end{tabular}

TABLEAU 1 : exemple de prononciation d'un échantillon de mots contenant la voyelle $<\mathrm{e}>$ inaccentuée à l'initiale de mot, selon les 3 dictionnaires de référence

\section{Méthode}

Afin de tester l'hypothèse ci-dessus, et de voir ce qu'il en est des productions des apprenants, nous avons effectué des mesures acoustiques automatiques sur les voyelles étudiées. Les 46 occurrences du corpus ont été mesurées chez nos 30 locuteurs (10 natifs, 10 apprenants indépendants et 10 apprenants expérimentés, voir 2.1) pour un total de 1325 voyelles après correction : quelques 
фccurrences ont dû être écartées car non réalisées comme December prononcé [t'sembə] ou erronées à cause d'une qualité de voix particulière par exemple). Le corpus a été phonétisé et aligné automatiquement avec SPPAS (Bigi, 2012), qui génère des fichiers TextGrid, puis corrigé manuellement sous PRAAT (Boersma, Weenink, 2001). Les mesures de 4 paramètres ont ensuite фté extraites pour chaque voyelle à l'aide d'un script PRAAT : F1, F2, F3 et durée. Les valeurs фorrespondent à la moyenne sur la durée de la voyelle. Les mesures ont ensuite été traitées dans NORM (Thomas, Kendall, 2007). Afin d'éviter les différences inter-locuteur résultant de la variation de longueur du conduit vocal, la procédure de normalisation Lovanov (1971) a été appliquée pour faciliter la comparabilité des données.

\section{Résultats}

\subsection{Analyse s formantiques}

\subsubsection{Locuteurs natifs}

Les résultats obtenus pour les valeurs formantiques chez les natifs sont montrés dans la figure 1. Les valeurs de F1 et F2 (en $\mathrm{Hz}$ ) sont données en ordonnée et en abscisse respectivement, ce qui permet ф’obtenir la représentation des voyelles sur un diagramme représentant l'espace vocalique (tronqué car les valeurs de F1 et F2 ont été ajustées pour plus de clarté sur la figure, ainsi tout l'espace łocalique n'est pas représenté ici). La valeur de F1 est corrélée avec le degré d'ouverture des voyelles (les valeurs basses correspondent à un faible degré d'aperture) et la valeur de F2 correspond à la position de la langue (réalisations antérieures vers la gauche, postérieures vers la droite). Les valeurs de F3 servent à la procédure de normalisation. On v oit clairement sur la figure 1 que la plupart des voyelles examinées sont concentrées vers la zone centrale haute, allant de 320 à $420 \mathrm{~Hz}$ pour $\mathrm{F} 1$ et de 1300 à $1700 \mathrm{~Hz}$ pour $\mathrm{F} 2$.

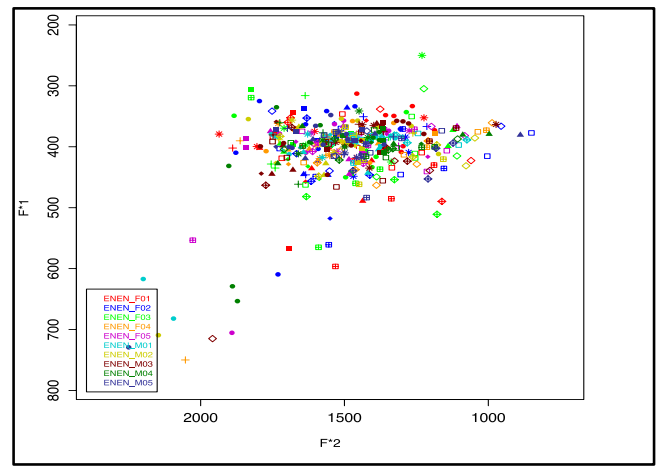

FIGURE 1: représentation dans NORM des valeurs normalisées de F1 et F2 pour les 413 voyelles étudiées et produites par les natifs

\subsubsection{Apprenants}

Les mêmes analyses formantiques ont été effectuées pour chacun des groupes d'apprenants (B et C) afin de les comparer à celles des natifs. Le but est d'obtenir une contribution pédagogique pour l'enseignement et l'apprentissage de l'anglais quant à la distribution et la production de cette voyelle. La figure 2 montre les résultats pour le groupe d'apprenants de niveau C. On constate des 
réalisations vocaliques très proches de celles des natifs anglais avec la production d'une voyelle inaccentuée centrale et haute, sensiblement plus haute que celle des natifs. La concentration des voyelles inaccentuées est légèrement plus éparse avec des valeurs allant de 300 à $400 \mathrm{~Hz}$ pour le F1 et de 1300 à $1700 \mathrm{~Hz}$ pour le F2. On note un nombre plus important de valeurs dissidentes (que nous nommerons par le terme anglais « outliers »).

Les résultats (figure 3) pour le groupe d'apprenants de niveau B diffèrent de façon remarquable. La figure montre des voyelles bien plus éparpillées avec des valeurs formantiques allant de 320 à 520 $\mathrm{Hz}$ pour le F1 et de 1400 à $2000 \mathrm{~Hz}$ pour le F2. Les voyelles sont plus antérieures et plus basses. Cette figure montre que ce groupe d'apprenants réduit beaucoup moins la voyelle inaccentuée $<\mathrm{e}>$ et ce pour la plupart des occurrences.

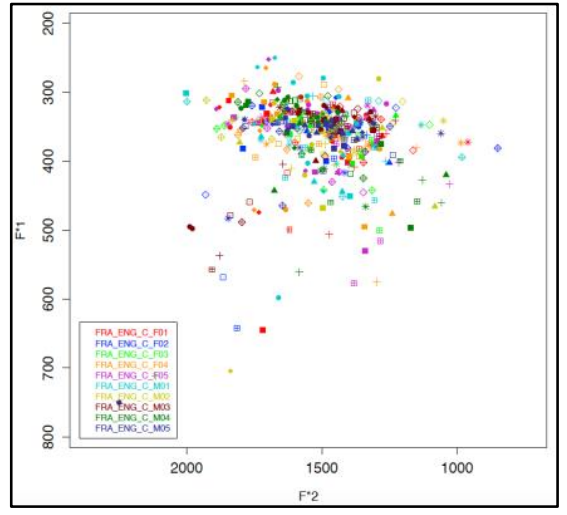

FIGURE 2 : valeurs normalisées F1 et $\mathrm{F} 2$, apprenants niveau $\mathrm{C}$

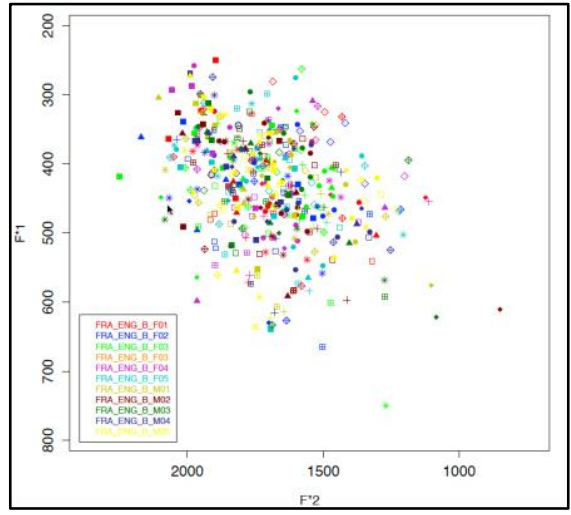

FIGURE 3 : valeurs normalisées F1 et F2, apprenants niveau B

Lorsque l'on compare les résultats des figures 1, 2 et 3, on observe une gradation des valeurs formantiques montrant ainsi les différences de niveau entre les deux groupes d'apprenants mais aussientre les apprenants et le groupe des natifs.

\subsubsection{Quelle tendance vocalique pour quel groupe?}

Nos valeurs étant normalisées, il s'avère difficile de les comparer avec les valeurs que l'on trouve dans la littérature. Nous avons donc pris les valeurs brutes et calculé les moyennes en séparant hommes et femmes : le tableau 2 ci-dessous donne les valeurs moyennes brutes de F1 et F2 pour/I/, /e/, / $/ /$ et /3:/ en discours suivi selon Deterding (1997), pour/i/ selon Herment, Turcsan (soumis), ainsi que les valeurs relevées dans notre corpus (deux dernières lignes).

Ces moyennes montrent que la voyelle type réalisée par nos natifs semble être une voyelle très haute, même légèrement plus haute que /I/. Il s'agit d'une voyelle centrale, plus centrale que /i/, et de façon très intéressante, on note que la valeur de $\mathrm{F} 2$ est très proche de celle donnée pour / $\mathrm{e} /$, cette dernière étant pourtant beaucoup plus basse. Cela veut dire que nous avons une voyelle centrale qui tend vers l'avant plutôt que vers l'arrière de l'espace vocalique. Il est difficile de dire s'il s'agit d'un schwa puisqu'il est connu que la qualité de schwa varie de façon conséquente selon les contextes (Browman, Goldstein, 1992, Bates, 1995, Flemming, 2009, Herry-Bénit et al., 2009, Heselwood, 2007, entre autres). Nos résultats confirment donc l'hypothèse de départ d'une centralisation en anglais RP contemporain de la voyelle $<\mathrm{e}>$ en position initiale inaccentuée. 
Les voyelles des apprenants du groupe $\mathrm{C}$ ont des valeurs proches de celles du [i], ce qui n'est pas vraiment surprenant puisque cette voyelle est proche de la voyelle française /i/. Les valeurs pour les apprenants de niveau B ne sont pas données car la déviation standard est trop importante, la moyenne n'est donc pas pertinente pourl'analyse.

\begin{tabular}{|c|c|c|c|c|}
\hline & \multicolumn{2}{|c|}{ Hommes } & \multicolumn{2}{|c|}{ Femmes } \\
\hline & F1 & F2 & F1 & F2 \\
\hline$/ \mathrm{I} /$ & 367 & 1757 & 384 & 2174 \\
\hline /e/ & 494 & 1650 & 719 & 2063 \\
\hline$/ \mho$ & 379 & 1173 & 410 & 1340 \\
\hline /3:/ & 478 & 1436 & 606 & 1695 \\
\hline [i] & 393 & 1962 & 406 & 2224 \\
\hline Natifs & 300 & 1634 & 374 & 1930 \\
\hline Apprenants C & 438 & 1816 & 453 & 2111 \\
\hline
\end{tabular}

TABLEAU 2 : valeurs moyennes brutes des voyelles hautes telles que trouvées dans la littérature et relevées dans notre corpus.

\subsection{Durées vocaliques}

La qualité de la voyelle réalisée est importante dans la perception du rythme en anglais, mais la quantité l'est également. Peterson, Barley (1952) expliquent qu'en anglais, en périphérie, en contexte inaccentué et à débit rapide, les voyelles ont tendance à être réduites en terme de durées car elles subissent une forte réduction vocalique. Nous pouvons donc prédire que, si les réductions vocaliques sont mal produites ou absentes chez les apprenants, les durées vocaliques des groupes d'apprenants seront plus importantes que celles des natifs. Les mesures de durées ont donc été relevées pour les voyelles examinées.

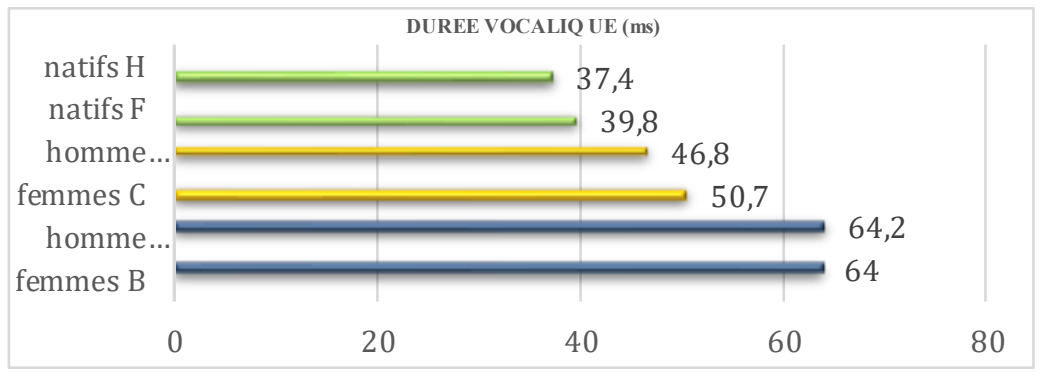

FIGURE 4: représentation des durées vocaliques des réalisations du corpus

La figure 4 regroupe la moyenne en millisecondes des productions pour chacun des groupes natifs, apprenants $\mathrm{C}$ et apprenants $\mathrm{B}$, hommes et femmes. On constate une nette gradation avec une augmentation des valeurs allant d'une moyenne de $38,6 \mathrm{~ms}$ pour les natifs, $48,75 \mathrm{~ms}$ pour les apprenants C, et jusqu'à $64,1 \mathrm{~ms}$ pour le groupe des apprenants B. Les productions du groupe d'apprenants indépendants (B) sont plus d'une fois et demi plus longues que celles des natifs. Les durées relevées pour les apprenants $\mathrm{C}$ montrent un léger allongement de la voyelle réduite. Cela corrobore les résultats trouvés lors des analyses formantiques, où nous avons expliqué que la figure 3 montre que les apprenants niveau $\mathrm{B}$ font beaucoup moins de réductions que les natifs et les apprenants C. Cela est probablement dû à la réalisation d'une voyelle française au lieu d'une réduction vocalique. La durée est donc un élément pertinent qui permet de distinguer les 3 différents groupes. Il est également intéressant de constater qu'en moyenne, les femmes font des voyelles 
légèrement plus longues que les hommes chez les natifs comme chez les apprenants $C$, ce qui va dans le sens de plusieurs études qui montrent que les durées vocaliques sont plus longues chez les femmes que les hommes dans plusieurs langues comme l'allemand, l'anglais ou le suédois (Hillenbrand et al., 1995, Simpson, 1998, Ericsdotter, Ericsson, 2001). Si nos apprenants de niveau B calquent des voyelles françaises sur les voyelles censées être réduites en anglais, ceci expliquerait alors pourquoi pour ce groupe la durée est quasiment la même entre hommes et femmes.

\section{Discussion et perspectives}

Les résultats obtenus permettent de dégager des éléments enrichissants concernant le processus allophonique. Les données formantiques sur l'analyse de la voyelle inaccentuée $<\mathrm{e}>$ mo ntrent que la prononciation tend vers une voyelle réduite centrale et haute en position initiale de mot et amènent à une neutralisation de l'opposition des voyelles /ə/, /I/ et /e/. Le fait que la voyelle schwa agisse comme une sorte de voyelle centrale variant en fonction du contexte n'est pas nouveau. En revanche, les résultats sur la prononciation de la voyelle inaccentuée $<\mathrm{e}>$ à l'initiale de mot apportés par les données dictionnairiques montrent un changement phonologique en cours : la tendance à la centralisation des voyelles réduites en anglais RP contemporain. Ils apportent aussi de nouveaux éléments pédagogiques quant à l'apprentissage de l'anglais : la possibilité de prononcer un /ə/pour tous les mots contenant la voyelle inaccentuée $<\mathrm{e}>$ à l'initiale, ce qui va permettre de faciliter les choses pour les apprenants, mais aussi pour les enseignants, qui se trouvent souvent bien embarrassés pour enseigner le phénomène complexe de la réduction ou non des voyelles inaccentuées en position initiale. L'analyse des durées vocaliques permet de mettre en avant la production d'un allongement vocalique et ce particulièrement dans les productions d'apprenants francophones de niveau B. Ce constat pourrait s'expliquer en menant une analyse perceptive qui confirmerait la réalisation d'une voyelle française et donc d'une trop grande tension. On pourrait étendre la comparais on aux durées syllabiques en prenant en compte la structure syllabique, les effets du contexte consonantique (Lindblom, 1963), les différences entre syllabes accentuées et inaccentuées (Gut, 2003), ainsi que le contexte prosodique. L'enjeu pédagogique est important car les résultats fournissent une aide précieuse quant à l'établissement de caractéristiques typologiques de strates d'interlangue. Une réplique de l'étude sur d'autres corpus tels que le corpus d'apprenants ANGLISH (Tortel, 2008) permettrait de confirmer ou infirmer cette possible "systématisation »de déviations caractéristiques d'un niveau de l'apprenant.

Notre recherche contribue à mieux comprendre la réalisation des voyelles inaccentuées chez les natifs anglophones et chez les apprenants, à poser une norme de référence pour la prononciation et à en établir les enjeux pédagogiques pourl'apprentissage et l'enseignement de l'anglais .

\section{Références}

BATES S. (1995). Towards a definition of schwa: an acoustic investigation of vowel reduction in English.Ph.D. dissertation, University of Edinburgh.

BIGI B. (2012). SPPAS: A Tool for the Phonetic Segmentation of Speech. In Proceedings of the Language Resource and Evaluation Conference. Istanbul: Turkey: 1748-1755.

BOERSMA P., WEENINK D. (2001). PRAAT, a system for doing phonetics by computer. Glot International 5/9-10: 341-345. http://www.praat.org, Version 5.4.04 
BoLINGER D. (1981). Two Kinds of Vowels, Two Kinds of Rhythm, Bloomington, IN, USA : IULC Publications, $68 \mathrm{p}$.

BROWMAN C., GOLDSTEIN L. (1992). “Targetless" schwa: an articulatory analysis. In LADD D.R. \& DOCHERTY G. (Eds.). Papers in Laboratory Phonology II. Cambridge: CUP, $26-67$.

Chan D., Fourcin A., Gibbon D., Grandström B., Huckvale M, Kokkinakis G., Kvale K., Lamel L., Lindberg B., Moreno A., Mouropoulos J., Senia F., Transcoso I., Velt C., ZEILIGER J. (1995). "EUROM - A Spoken Language Ressource for the EU." In Proceedings of Eurospeech'95, Madrid.

Deterding D. (1997). The formants of monophthong vowels in Standard Southern British English pronunciation. Journal of the International Phonetic Association 27, 47-55.

ERICSDOTTER C., ERICSSON A.M. (2001). Gender differences in vowel duration in read Swedish: Preliminary results. In Proc. Fonetik 2001, XIVth Swedish Phonetics Conference. Working Papers of the Department of Linguistics Volume 49. Lund University, 34-37.

FLEMming E. (2009). The phonetics of schwa vowels, in MinOva D. (Ed.). Phonological Weakness in English. Houndsville : Palgrave Macmillan, 78-95.

GENDROT C., ADDA-DECKER M. (2007). Impact of duration and vowel inventory size on Formant yalues of aeral vowels: an automated formant analysis from eight languages. In: International Congress of Phonetics Sciences, pp.1417-1420, Germany.

GUT U. (2003) Non-native Speech rhythm in German. Proceedings of $15^{\text {th }}$ International Congress of Phonetic Sciences, Barcelona. 2437-2440.

GUT U. (2009). Non-native Speech. A corpus-based Analysis of Phonological and phonetic Properties of L2 English and German, Frankfurt: Peter Lang.

HERMENT S. (2010). The pedagogical implications of variability in transcription, the case of [i] and [u]. In Henderson A. (Ed.). English Pronunciation: Issues and Practices (EPIP): Proceedings of the First International Conference. Université de Savoie, Chambéry, France, 177-188.

HERMENT S., LOUKINA A., TORTEL A. (2012). AIXOX. SLDR, http://sldr.org/sldr000784/fr

Herment S., Tortel A., Bigi B., Hirst D., Loukina A. (2014). AixOx, a multi-layered learners' corpus: automatic annotation. In DÍAZ PÉREZ J., DÍAZ NEGRILlO A. (Eds.). Specialisation and variation in language corpora. Bern: Peter Lang, 41-76.

HERMENT S., TURCSAN G. (Soumis). Revisiting contrasts for high vowels in English: Allophony, Neutralisation and Phonological corpora.

Herry-Benit N., Nikolov R., TORTEl A. (2009). Positional determination of the quality of schwa in English. Scientific Works-Philology, vol. 47, Book 1 Part B. Plovdiv University, Bulgaria, 247257. 
HESELWOOD B. (2007). Schwa and the Phonotactics of RP English. Transactions of the Philological Society, vol. 105, no. 2.

Hillenbrand J., Getty L.A, Clark M.J., Wheeler K. (1995). Acoustic characteristics of American English vowels. Journal of the Acoustical Society of America 97, 3099-3111.

HIRST et al. (2013). Building OMProDat: an open multilingual prosodic database. Proceedings of TRASP, September 2013, Aix-en-Provence, France, 11-14. http://sldr.org/sldr000725

HORGUES C. (2010). Prosodie de l'accent français en anglais et perception par des auditeurs anglophonesnatifs. Thèse de doctorat, Université Paris Diderot-Paris 7.

JONES D. (1967). Everyman's English Pronouncing Dictionary, $13^{\text {th }}$ ed. London: J.M. Dent \& Sons.

JONES D. (2011). English Pronouncing Dictionary, 18th edition (revised by RoACH P., SETTER J., ESLING J.). Cambridge: CUP.

LiNDBLOM B. (1963). On vowel reduction, Report \#29, The Royal Ins. of Tech., Speech Transmission Laboratory, Stockholm, Sweden.

LOBANOV B.M. (1971). Classification of Russian vowels spoken by different listeners. JASA 49, 606-08.

Peterson G., BARLey H. (1952). Control Methods Used in a Study of the Vowels, Journal Acoustical Society of America, Vol. 24: 175-184.

SIMPSON A.P. (1998). Phonetische Datenbanken des Deutschen in der empirischen Sprachforschung und der phonologischen Theoriebildung. Arbeitsberichte des Instituts für Phonetik und digitale Sprachverar-beitung der Universität Kiel (AIPUK) 33.

ThOMAs E.R., Kendall T. (2007). NORM: The vowel normalization and plotting suite. [Online Resource: http://ncslaap.lib.ncsu.edu/tools/norm/ ]

TORTEL A. (2008). ANGLISH : base de données comparatives $11 \& 12$ de l'anglais lu, répété et parlé. Travaux Interdisciplinaires du Laboratoire Parole et Langage (TIPA), 27, 111-122.

TORTEL A. (2009). Evaluation qualitative de la prosodie d'apprenants français : apport de paramétrisations prosodiques. Thèse de doctorat, Aix-Marseille Université.

WELLSJ.C. (2008). Longman Pronunciation Dictionary, 3rd edition. London: Longman.

WENK B.J., WiOLAND F. (1982). Is French really syllable-timed? Journal of Phonetics, 10, 193-216. 